\title{
Avaliação do tratamento da hérnia perineal bilateral no cão por acesso dorsal ao ânus
}

\section{Evaluation of surgical treatment in dogs with bilateral perineal hernia by dorsal anus access}

\author{
Andrea ACAUI ${ }^{1}$; Angelo João STOPIGLIA ${ }^{1}$; Julia Maria MATERA ${ }^{1}$; Silvia Renata Gaido \\ CORTOPASSI ${ }^{1}$; Procássia Maria de Oliveira LACERDA ${ }^{1}$
}

${ }^{1}$ Departamento de Cirurgia da Faculdade de Medicina Veterinária e Zootecnia da Universidade de São Paulo, São Paulo-SP

\begin{abstract}
Resumo
O objetivo do presente estudo foi avaliar a evolução e as complicações pós-operatórias do tratamento de hérnias perineais bilaterais, por acesso dorsal ao ânus, em 14 cães com idade entre seis e 12 anos. Realizou-se incisão em forma de 'U' invertido, a qual se estendeu desde a porção lateral da tuberosidade isquiática do lado esquerdo, curvando-se em direção à linha mediana, dois centímetros dorsalmente ao ânus e dirigindo-se simetricamente para a extremidade da tuberosidade isquiática contralateral. Após redução da hérnia, os músculos do diafragma pélvico direito e esquerdo foram suturados pela técnica de elevação do músculo obturador interno. As complicações observadas no período pósoperatório foram incontinência fecal, contaminação da ferida, deiscência de pontos da sutura cutânea, incontinência urinária e recidiva em um cão 19 dias após procedimento cirúrgico. O acesso utilizado foi exequível e todos os cães submetidos à técnica operatória proposta não apresentaram recidivas em um período entre cinco e dez meses de avaliação pós-operatório.
\end{abstract}

Palavras-chave: Cães. Cirurgia. Hérnia perineal.

\begin{abstract}
Observing the evolution and complications in the treatment of bilateral perineal hernias by dorsal access to the anus was the objective of this study. Fourteen dogs of various breeds were operated with an inverted " $U$ " shaped incision, which extended from the lateral portion of the tuberosity Isquiatica on the left, bending into the direction of the midline, dorsally to anus and heading out symmetrically to the tip of the tuberosity Isquiatica contralateral. After reduction of the hernia, the right and left muscles of the pelvic diaphragm were sutured by the technique of raising the internal obturator muscle. The complications observed in the postoperative period were fecal incontinence, contamination of the wound, dehiscence of suture points of the skin, urinary incontinence and recurrence in a dog with 19 days after surgery. The access used was feasible and all dogs undergoing surgical technical proposal showed no recurrence in a period of five to ten months of postoperative assessment.
\end{abstract}

Keywords: Dogs. Surgery. Perineal hernia.

\section{Introdução}

A hérnia perineal é caracterizada por acometer quase que exclusivamente cães machos e idosos, sendo motivo de estudo por parte de pesquisadores há décadas, devido ao fato de sua etiologia não estar ainda totalmente esclarecida ${ }^{1}$.

A hérnia perineal é frequentemente encontrada em cães machos com sete a nove anos de idade e as raças com maior predisposição são: Boston terrier, Collie, Pequinês, Old English Sheepdog, Pastor Alemão, Dachshund e Boxer, assim como os cães sem raça definida ${ }^{1}$.

Nas cadelas, a hérnia perineal normalmente não ocorre, pois o músculo elevador do ânus é mais po- tente, largo e espesso, com maior área de contato com a parede retal do que no macho ${ }^{2}$.

A deterioração da função de sustentação do diafragma pélvico é considerada parte integral ou précondição no desenvolvimento da hérnia perineal e, na

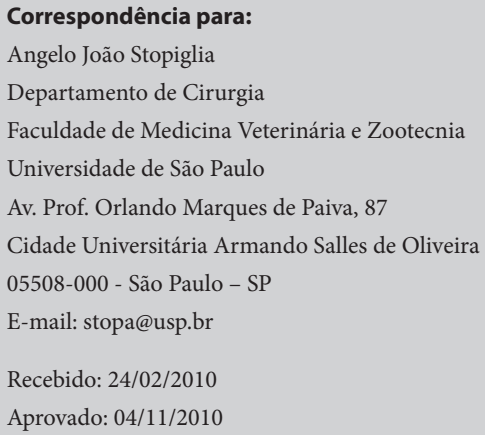


maioria dos casos, o músculo elevador do ânus está atrofiado ou até ausente ${ }^{3,4}$. A fragilidade do diafragma pélvico frequentemente leva a desvio lateral da porção final do reto ipsis lateral a hérnia, e normalmente há acúmulo de fezes não evacuada, apesar das tentativas do animal em defecar" ${ }^{4}$.

A gordura retroperitoneal, localizada no canal pélvico, constitui componente principal do conteúdo herniado ${ }^{5}$, porém, flexura ou saculação retal, próstata, líquido, tecido conjuntivo, bexiga urinária e intestino delgado podem estar presentes ${ }^{6}$.

Muitos métodos têm sido descritos para o reparo das hérnias perineais nos cães. A partir da técnica clássica ${ }^{6}$, que sutura o músculo coccígeo ao esfíncter anal externo, desenvolveram-se diversas modificações para diminuir a incidência das recidivas. Dentre elas, citam-se a elevação do músculo obturador interno $^{7,8,9}$ e suas modificações ${ }^{10}$, a transposição do músculo glúteo superficial ${ }^{11}$, além da correção cirúrgica do diafragma pélvico por meio de sua obliteração mediante adaptação de estruturas sintéticas como malha de polipropileno ${ }^{12,13}$, ou membranas biológicas como centro frênico equino ${ }^{14}$ e colágeno dérmico suíno ${ }^{15}$, pericárdio equino ${ }^{16}$ e peritônio de bovino ${ }^{17}$.

Adicionalmente, o emprego do músculo semitendinoso tem sido preconizado como restaurador do diafragma pélvico ${ }^{18}$. Mckeown et al. ${ }^{19}$ sugerem a correção simultânea das hérnias perineais bilaterais, entre outras afecções, por meio de uma abordagem cirúrgica dorsal a região perineal e reto de cães. Em estudo com cães sadios, estes autores avaliaram esta via de acesso cirúrgico e não observaram complicações como incontinência fecal ou desconforto na evacuação no período de três semanas pós-operatório.

O objetivo do estudo foi testar, em cães com hérnia perineal, o acesso dorsal ao ânus com incisão única, como via para a correção da afecção bilateral em tempo operatório único, observando-se a evolução e complicações pós-operatórias.

\section{Material e Método}

Foram utilizados 14 cães machos de diferentes raças e sem raça definida, com idade entre seis e 12 anos, peso variando entre 4,2 e 40,0 kg, portadores de hérnia perineal bilateral, triados no Hospital Veterinário (HOVET) da Faculdade de Medicina Veterinária e Zootecnia da Universidade de São Paulo e encaminhados ao Serviço de Cirurgia de Pequenos Animais do Departamento de Cirurgia da mesma faculdade, no período de setembro de 2000 a janeiro de 2001.

Todos os pacientes foram avaliados clinicamente quanto ao tempo de evolução das hérnias perineais bilaterais, desde o momento em que o proprietário observou o aumento de volume na região de períneo até o atendimento no HOVET. A palpação digital externa da fossa ísqueo-retal e o toque retal foram utilizados para confirmar o diagnóstico das hérnias perineais e auxiliar na avaliação do conteúdo provável herniado. Solicitou-se exame ultrassonográfico, avaliação da função cardíaca e análise sérica de ureia, creatinina, hematócrito e proteína total.

Os pacientes foram submetidos a jejum hídrico e alimentar de 12 horas e terapia com antibióticos a base de ampicilina (22,0 mg/kg/TID) e metronidazol (15,0 mg/kg/TID), 48 horas antes do ato operatório. Os cães receberam numeração de 1 a 14, em ordem cronológica com o ato operatório. Fixou-se sonda trans-uretral apropriada, quando em caso de anúria.

Os cães foram posicionados em decúbito esternal. A mesa cirúrgica foi colocada em posição de Trendelenburger a fim de elevar a pelve do animal. Todos os cães permaneceram com sonda uretral durante o ato operatório. Foi realizada incisão em forma de "U" invertido (Figura 1a), a qual se estendeu desde a porção lateral da tuberosidade isquiática do lado esquerdo, curvando-se em direção dorsal à linha mediana, passando dois centímetros dorsalmente ao ânus, e dirigindo-se simetricamente para a extremidade lateral da tuberosidade isquiática contralateral. 
O músculo retococcígeo foi preservado, enquanto os sacos herniários foram delicadamente rompidos através de divulsão digital romba (Figura 1b). As vísceras foram reduzidas à cavidade pélvica e abdominal e os músculos esfíncter anal externo, coccígeo, obturador interno, elevador do ânus e o reto foram identificados. Avaliou-se o grau de atrofia dos referidos músculos, de acordo com os seguintes parâmetros: não atrofiado; atrofia moderada; visível, bastante atrofiado e não visibilizado. Para a correção cirúrgica das hérnias perineais foi escolhida a técnica da elevação do músculo obturador interno, descrita por Early e Kolata ${ }^{7}$. O tecido subcutâneo foi aproximado por sutura contínua com poliglactina 910 número 2-0 e a pele com fio de náilon monofilamento número $2-0$ por meio de pon- tos simples e separados (Figura 1c). Todos os cães não castrados foram submetidos à orquiectomia por incisão pré-escrotal por meio da técnica clássica.

Os pacientes operados receberam antibióticos (metronidazol - $15 \mathrm{mg} / \mathrm{kg} / \mathrm{BID}$ e ampicilina - 22,0 mg/ $\mathrm{kg} / \mathrm{TID}$ ) por dez dias, associados com cetoprofeno na dose de $1 \mathrm{mg} / \mathrm{kg}$, por quatro dias.

A avaliação pós-operatória foi feita por observação clínica da região perineal e toque retal, nos períodos de 48 horas, sete dias, e 15 dias após o procedimento cirúrgico. Posteriormente, em período que variou entre cinco e dez meses de pós-operatório os animais foram avaliados sobre o estado geral, presença de incontinência fecal, presença de aumento de volume perineal e disquesia.

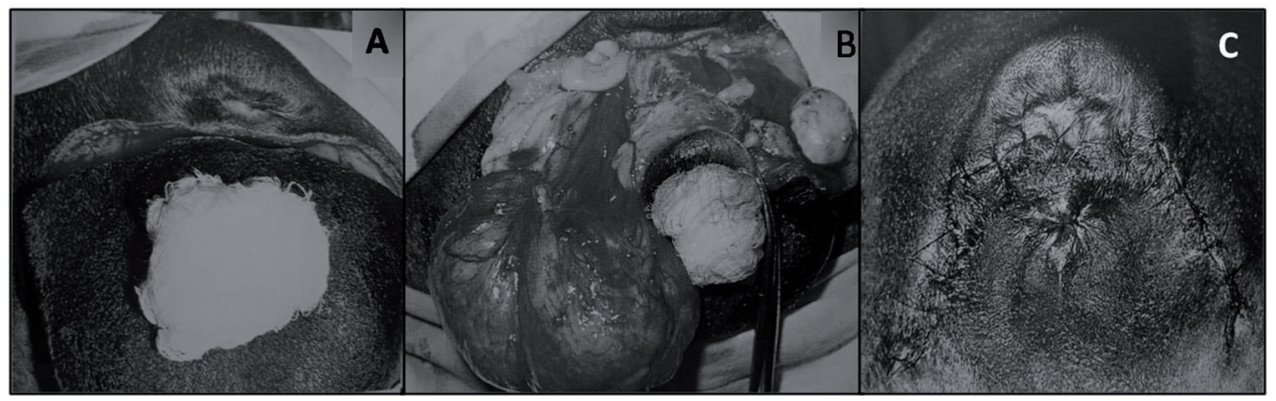

Figura 1 - Correção cirúrgica de hérnia perineal bilateral por acesso dorsal ao ânus. a: Incisão dorsal ao ânus (cão 13), b: Exposição do saco herniário (cão 13), c: Formato da incisão após correção (cão 04)

\section{Resultados}

A idade dos pacientes variou de seis anos e seis meses a 12 anos e seis meses (média de nove anos) e o peso entre 4,2 e $40 \mathrm{~kg}$. Os cães distribuíram-se em sem raça definida $(n=4)$, Boxer $(n=2)$, Old English Sheepdog $(\mathrm{n}=2)$, Poodle $(\mathrm{n}=1)$, Pastor Alemão $(\mathrm{n}=$ $1)$, Collie $(n=1)$, Pinscher $(n=1)$, Rottweiler $(n=1)$ e Weimaraner $(\mathrm{n}=1)$, conforme quadro 1 .

O tempo de evolução de surgimento das hérnias variou de três até 36 meses. Apenas o paciente $n^{\circ} 02$ já recebera tratamento cirúrgico há três anos, com recidiva de um ano. Ao exame clínico, os animais apre- sentaram aumento de volume perineal que variou entre unilateral e bilateral. $\mathrm{O}$ período transoperatório transcorreu sem intercorrências. A figura 2 mostra os diferentes graus de atrofia da musculatura que compõe a fossa isquiorretal.

A variação das complicações pós-operatórias observadas até o momento da retirada da sutura cutânea, assim como no período pós-operatório tardio, podem ser observadas na figura 3. Considerando o último exame de toque retal, constatou-se integridade dos diafragmas pélvicos direito e esquerdo, exceto o cão número 02 que apresentou ruptura do diafragma 


\begin{tabular}{|c|c|c|c|c|}
\hline Cão & Raça & Idade & Peso $(\mathrm{kg})$ & Tempo de evolução (meses) \\
\hline 1 & Boxer & 7 anos, 2 meses & 30,0 & 5 \\
\hline 2 & Pastor Alemão & 11 anos, 3 meses & 38,0 & 36 \\
\hline 3 & S.R.D. & 11 anos & 20,8 & 6 \\
\hline 4 & Weimaraner & 8 anos, 7 meses & 34,2 & 12 \\
\hline 5 & Sheepdog & 8 anos & 34,0 & 24 \\
\hline 6 & Sheepdog & 12 anos, 10 meses & 40,0 & 2 \\
\hline 7 & S.R.D. & 8 anos & 23,0 & 2 \\
\hline 8 & S.R.D. & 9 anos & 20,0 & 3 \\
\hline 9 & Pinscher & 10 anos, 8 meses & 4,2 & 3 \\
\hline 10 & Collie & 10 anos & 20,3 & 7 \\
\hline 11 & Rottweiler & 9 anos, 7 meses & 35,0 & 12 \\
\hline 12 & S.R.D. & 6 anos, 6 meses & 19,3 & 24 \\
\hline 13 & Poodle & 11 anos, 3 meses & 6,4 & 36 \\
\hline 14 & Boxer & 7 anos, 7 meses & 39,0 & \\
\hline
\end{tabular}

Quadro 1 - Características dos cães submetidos a tratamento cirúrgico de Hérnia perineal bilateral por acesso dorsal ao ânus em tempo cirúrgico único, avaliados segundo raça, idade, peso e tempo de evolução $(\mathrm{kg}=$ quilograma; $\mathrm{SRD}=$ sem raça definida $)$
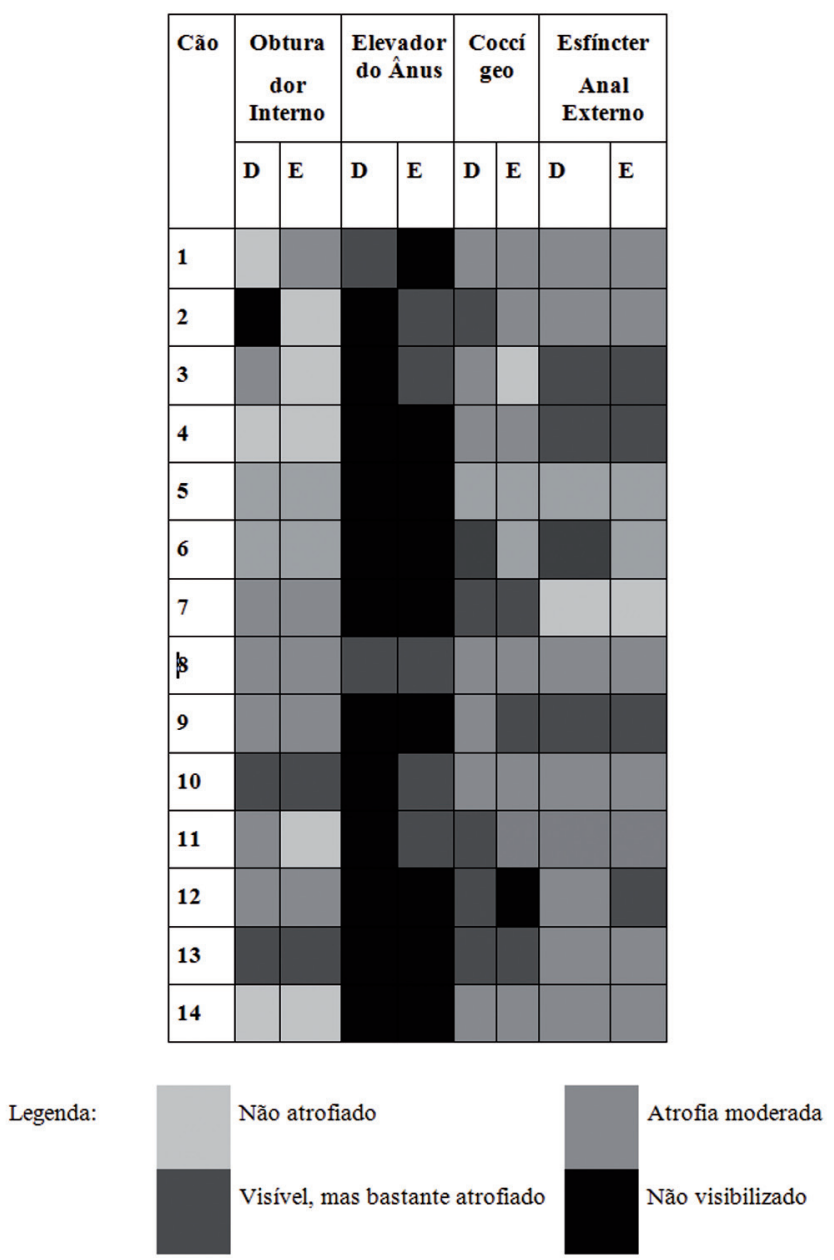

Figura 2 - Quadro demonstrativo dos diferentes graus de atrofia da musculatura da fossa isquiorretal observadas nos cães operados 

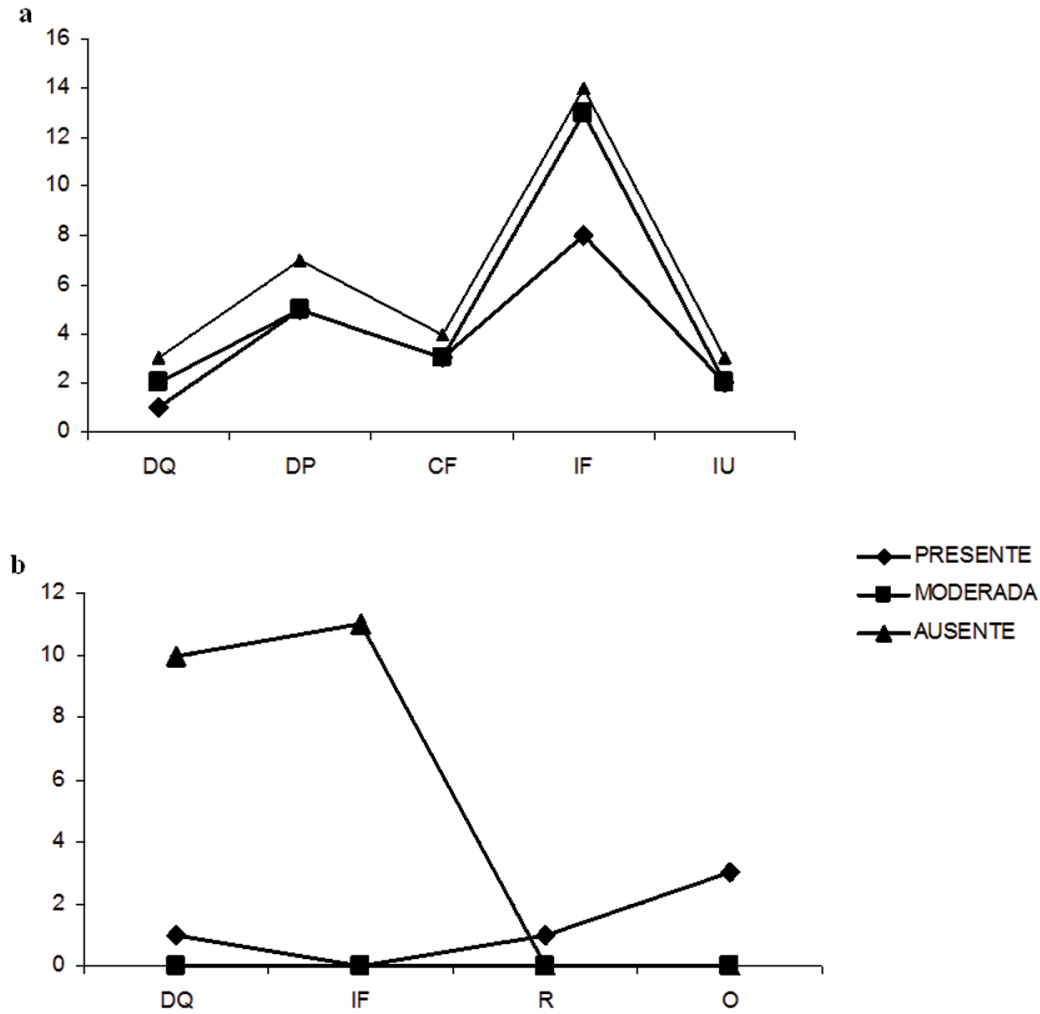

Figura 3 - Demonstração gráfica das complicações pós-operatórias observadas antes da retirada dos pontos até dezenove dias (a) e no pós-operatório tardio com até dez meses (b). $\mathrm{DQ}=$ Disquesia, $\mathrm{DP}=$ Deiscência de Pele, $\mathrm{CF}=$ Contaminação da Ferida, IF = Incontinência Fecal, IU = Incontinência Urinária, $\mathrm{R}=$ Recidiva, $\mathrm{O}$ = Óbito

pélvico direito 19 dias após procedimento cirúrgico, sendo sacrificado após três meses.

Os cães números 02, 04, 08, 09, 12 e 13 apresentaram desvio ventral de reto. No caso ${ }^{\circ}{ }^{04}$, houve necessidade de manejo alimentar, laxantes e enema para evitar tenesmo e constipação seis meses após procedimento cirúrgico.

A incontinência fecal foi temporária, e todos os cães voltaram a ter controle da evacuação no período pósoperatório tardio (Figura 3). O cão $n^{\circ} 06$ foi a óbito no quarto dia de pós-operatório, o mesmo evacuava e urinava normalmente, porém não conseguia ficar em estação.

\section{Discussão}

A herniorrafia bilateral em tempo operatório único foi estudada por vários pesquisadores ${ }^{6,10,20,21,22}$, porém ainda há controvérsia no que tange à comparação deste tipo de correção cirúrgica com a correção em dois tempos.

Quando corrigida em apenas um tempo operatório, a literatura aborda esta paratopia através de incisão cutânea que se estende lateralmente ao ânus desde a base da cauda até a tuberosidade isquiática ${ }^{6}$, exceto na técnica de transposição do músculo glúteo superficial ${ }^{11}$. $\mathrm{O}$ acesso dorsal ao ânus, com incisão única, constitui uma opção às técnicas já estabelecidas.

A idade e o sexo dos animais operados estavam dentro do padrão esperado, ou seja, ocorrem principalmente ${ }^{10} \mathrm{em}$ cães machos a partir de cinco anos de idade $\mathrm{i}^{1,3,6,7,22}$.

O comprometimento da musculatura da região de períneo, provocado por atrofia dos músculos que compõem o diafragma pélvico, foi avaliada macros- 
copicamente, obtendo graus variados de atrofia. Em toda literatura que cita a hérnia perineal essa atrofia é mencionada ${ }^{4,10}$, sendo o músculo elevador do ânus o mais severamente atrofiado e frequentemente não visibilizado ${ }^{4}$. Já o músculo coccígeo e o esfíncter anal externo apresentam, tipicamente, graus de atrofia moderada. Esta mesma avaliação macroscópica foi constatada nos animais submetidos à técnica proposta.

Apesar de Orsher ${ }^{9}$, Bilbrey, Smeak e De Hoff ${ }^{23}$ e Dupré, Prat e Bouvy ${ }^{24}$ relatarem uma maior atrofia muscular quanto maior o tempo de evolução em hérnias perineais bilaterais, verificou-se em alguns casos no presente trabalho, graus de atrofia consideráveis mesmo nos animais com tempo de evolução mais curto. Cabe ressaltar, no entanto, que o tempo de evolução é um dado subjetivo e sujeito a imprecisões.

A incontinência fecal foi a complicação pós-operatória mais frequente, o mesmo verificado por Van Sluis e Sjollema ${ }^{10}$, que observaram tal sintoma em $25 \%$ dos cães submetidos a herniorrafia bilateral. No entanto, conforme a literatura informa, o controle da evacuação foi recuperado até 30 dias do ato operatório $^{3,7,21}$. Van Sluis e Sjollema ${ }^{10}$ cogitam a possibilidade da incontinência fecal ser mais uma complicação da hérnia do que do próprio ato operatório, já que metade dos cães apresentava incontinência fecal antes de operados. Mckeown et al. ${ }^{19}$ utilizaram a via de acesso dorsal ao ânus em cães saudáveis e não tiveram como complicação o quadro de incontinência fecal. Van Sluis e Sjollema ${ }^{10}$ consideram que compressões repetidas e o estiramento do nervo pudendo durante o esforço na evacuação, podem eventualmente resultar em danos aos nervos e deterioração da função do esfíncter.

Segundo Matthiesen ${ }^{25}$, durante a dissecção podem ocorrer danos aos nervos pudendo e retal caudal e ao músculo esfíncter anal externo. Considerando que o acesso dorsal ao ânus, expõe pelo menos dois terços da superfície deste músculo, é razoável admitir que os riscos de trauma sejam maiores, logo a técnica pro- posta deve ser realizada com maior diligencia comparando as técnicas usuais de correção.

Observou-se incontinência urinária transitória em um dos dois casos de retroflexão de bexiga relatados neste estudo. Segundo Matthiesen ${ }^{25}$ e Bilbrey, Smeak e De Hoff ${ }^{23}$, cães com retroflexão da bexiga podem desenvolver atonia ou incontinência urinária transitórias ou permanentes, dependendo da gravidade da lesão dos vasos e nervos e da duração do encarceramento.

A herniorrafia bilateral, além de favorecer tenesmo por dor e possibilitar a aposição de pontos de sutura no lúmen retal são relatados como possíveis fatores predisponentes ao prolapso retal ${ }^{25}$, devido, por certo, à estimulação contínua do plexo nervoso intramural local. No entanto, não foi observada tal complicação em nenhum dos cães deste estudo apesar de submetidos a herniorrafia bilateral em tempo único.

No presente estudo apenas um cão, o número 02, teve recidiva da hérnia direita 19 dias após o procedimento cirúrgico, sendo que o mesmo fora submetido a herniorrafia prévia, corroborando com Burrows e Harvey $^{21}$ que registraram maiores riscos de recidiva em animais submetidos a herniorrafias repetidas. Este resultado é comparável ao de Van Sluis e Sjollema ${ }^{10}$, onde das cinco recidivas dos 103 cães operados, duas ocorreram após herniorrafia bilateral, uma após herniorrafia unilateral e duas após remoção de sutura profunda por fistula perineal, sendo que as três primeiras recidivaram em quatro, oito e 14 semanas de pós-operatório.

As recidivas em tempos superiores a um ano estão associadas à contínua deterioração do tecido perineal ${ }^{4}$. Como o tempo de observação do pós-operatório deste trabalho variou de cinco a dez meses não há como confrontar os dados com o citado acima. No entanto, pode-se afirmar, assim como Bellenger ${ }^{21}$ que tecnicamente o procedimento proposto foi adequado. O óbito, observado no animal número 06 , tem como fatores concorrentes o 
peso elevado $(40,0 \mathrm{~kg})$, que pode ter interferido na recuperação pós-operatória da anestesia epidural, associada à idade do animal (superior a 12 anos) e raça, além de a hérnia estar presente há 24 meses. Quanto às demais intercorrências pós-operatórias observadas, em face de ser quadro de hérnia perineal bilateral, todas são compatíveis com o relatado na literatura compulsada ${ }^{1,2,3,4}$.

As herniorrafias de forma geral requerem pós-operatórios cautelosos, sendo o proprietário peça essencial ao sucesso da correção cirúrgica. Por isso, a correção cirúrgica bilateral em tempo cirúrgico único por acesso dorsal ao ânus possibilitou ao proprietário cuidar de seu animal em apenas um período pós-ope-

\section{Referências}

1. WEAVER, A. D.; OMAMEGBE, J. O. Surgical treatment of perineal hernia in the dog. Journal of Small Animal Practice, v. 22 , p. $749-758,1981$.

2. DESAI, R. An anatomical study of the canine male and female pelvic diaphragm and the effect of testosterone on the status of levator ani of male dogs. Journal of the American Animal Hospital Association, v. 18, p. 195-202, 1982.

3. HARVEY, C. E. Treatment of perineal hernia in the dog - a reassessment. Journal of Small Animal Practice, v. 18, p. 505$511,1977$.

4.BELLENGER, C. R.; CANFIELD, R. B. Hernia perineal. In: SLATTER, D. Manual de cirurgia de pequenos animais. 2. ed. São Paulo: Manole, 1998. p. 578-590.

5.LIPOWITZ, A. J. Perineal Surgery. In: LIPOWITZ, A. J.; CAYWOOD, D. D.; NEWTON, C. D.; SCHWARTZ, A Complications in small animal surgery. Baltimore: Williams \& Wilkins, 1996. p. 529-536.

6. PETTIT, G. D. Perineal hernia in the dog. Cornell Veterinary, v. 52, p. 261-279, 1962.

7.EARLEY, D. T.; KOLATA, R. J. Perineal hernia in the dog: an alternative method of correction. In: BOJRAB, M. J. Current techniques in small animal surgery. Philadelphia: Lea \& Febiger, 1983. p. 405-407.

8. ORSHER, R. J.; JOHNSTON, D. E. The surgical treatment of perineal hernia in dogs by transposition of the obturator muscle. Compendium of Continuing Education Practicing Veterinarian, v. 7, p. 233-239, 1985.

9. ORSHER, R. J. Clinical and surgical parameters in dogs with perineal hernia - Analysis of results of internal obturator transposition. Veterinary Surgery, v. 15, n. 3, p. 253-258, 1986.

10. VAN SLUIS, F. J.; SJOLLEMA, B. E. Perineal hernia repair in the dog by transposition of the internal obturator muscle -I e II. Complications and results in 100 patients. The Veterinary Quarterly, v. 11, n. 1, p. 12-23, 1989. ratório, aumentando seu conforto, diminuindo assim seus custos com medicamentos e evitando custos com o segundo procedimento cirúrgico.

\section{Conclusão}

A técnica proposta mostrou-se de fácil execução e possibilitou boa visualização das estruturas que compõem as fossas isquiorretais por aumentar o campo operatório. A evolução e complicações pós-operatórias não fogem ao mencionado na literatura, por tanto, o acesso dorsal ao ânus constitui-se uma alternativa na correção cirúrgica de hérnia perineal bilateral em tempo operatório único, tornando-se uma técnica vantajosa para paciente e proprietário.

11.SPREULL, J. S. A.; FRANKLAND, A. L. Transplanting the superficial gluteal muscle in the treatment of perineal hernia and flexure of the rectum in the dog. Journal of Small Animal Practice, v. 21, p. 265-278, 1980.

12.MATERA, A.; BARROS, P. S. M.; STOPIGLIA, A. J.; RANDI, R. E. Hérnia perineal no cão - Tratamento cirúrgico mediante utilização de malha de polipropileno. Revista da Faculdade de Medicina Veterinária da Universidade de São Paulo, v. 18, n. 1, p. 37-41, 1981.

13. CLARKE, R. E. Perineal herniorrhaphy in the dog using polypropylene mesh. Australian Veterinary Pratice, v. 19, n. 1, p. 8-14, 1989.

14.ALVARENGA, J.; BARROS, P. S. M.; STOPIGLIA, A. J.; IWASAKI, M. Hérnia perineal no cão (Canis familiaris) Tratamento cirúrgico mediante implante de centro frênico em glicerina. In: CONFERÊNCIA ANUAL DA SOCIEDADE PAULISTA DE MEDICINA VETERINÁRIA, 25., 1978, São Paulo. Resumo... 1978.

15.FRANKLAND, A. L. Use of porcine dermal collagen in the repair of perineal hernia in dogs - a preliminary report. Veterinary Record, v. 119, p. 13-14, 1986.

16.ZERWES, M. B. C. Avaliação comparativa do tratamento cirúrgico da hérnia perineal em cães pela técnica de elevação do músculo obturador interno com ou sem reforço de retalho de membrana de pericárdio equino preservado em glicerina a 98\%. 2005. 91 p. Dissertação (Mestrado) Faculdade de Medicina Veterinária e Zootecnia, Universidade de São Paulo, São Paulo, 2005.

17.DALECK, C. R.; DALECK, C. L. M.; FILHO, J. G. P.; NETO, J. M. C. Reparação da hérnia perineal em cães com peritônio de bovino conservado em glicerina. Ciência Rural, v. 22, n. 2, p. 179-183, 1992.

18.MORTARI, A. C.; RAHAL, S. C.; RESENDE, L. A. L.; DAL-PAISILVA, M.; MAMPRIM, M. J.; CORREA, M. A.; ANTUNES, S. H. S.; HAYES, H. W.; WILSON, G. P.; TRONE, R. E. Electromyographical, ultrasonographical and morphological 
modifications in semitendinous muscle after transposition as ventral perineal muscle flap. Journal Veterinary Medicine, v. 52, n. 7, p. 359-365, 2005.

19. MCKEOWN, D. B.; COCKSHUTT, J. R.; PARTLOW, G. D.; DE KLEER, V. S. Dorsal approach to the caudal pelvic canal and rectum - Effect on normal dogs. Veterinary Surgery, v. 13, n. 13, p. 181-184, 1984.

20.BURROWS, C. F.; HARVEY, C. E. Perineal hernia in the dog. Journal of Small Animal Practice, v. 14, p. 315-322, 1973.

21.BELlenger, C. R. Perineal hernia in dogs. Australian Veterinary Journal, v. 56, p. 434-438, 1980.

22.HOSGOOD, G.; HEDLUND, C. S.; PECHMAN, R. D.; DEAN, P. W. Perineal herniorrhaphy: perioperative data from 100 dogs. Journal of the American Animal Hospital Association, v. 31, p. 331-342, 1995.

23. BILBREY, S. A.; SMEAK, D. D.; DE HOFF, W. Fixation of the deferent ducts for retrodisplacement of the urinary bladder and prostate in canine perineal hernia. Veterinary Surgery, v. 19, n. 1, p. 24-27, 1990.

24.DUPRÉ, G. P.; PRAT, N.; BOUVY, B. Perineal hernia in the dog: evaluation of associated lesions and results in 60 dogs. Abstract of the European College of Veterinary Surgeons. Veterinary Surgery, v. 22, p. 250, 1993.

25.MATTHIESEN, D. T. Diagnosis and management of complications occurring after perineal herniorrhaphy in dogs. Compendium of Continuing Education Practicing Veterinarian, v. 11, n. 7, p. 797, 1989. 